\title{
Conclusiones del Simposium 2007 de la Sociedad Española de Medicina Oral sobre "Xerostomía. Síndrome de Boca Seca. Boca Ardiente"
}

Bascones $\mathrm{A}^{1}$, Tenovuo $\mathrm{J}^{2}$, Ship $\mathrm{J}^{3}$, Turner $\mathrm{M}^{4}$, Mac-Veigh ${ }^{5}$, López-lbor $\mathrm{JM}^{6}$, Albi $\mathrm{M}^{7}$, Lanzós $\mathrm{E}^{8}$, Aliaga $\mathrm{A}^{9}$

\section{INTRODUCCIÓN}

El Profesor Antonio Bascones, presidente de la Sociedad Española de Medicina Oral, marcó durante su introducción la importancia y las directrices a seguir en la Xerostomía o hiposialia.

La saliva es esencial para mantener una boca saludable; por lo que la falta de secreción de las glándulas salivales es un problema importante de salud, debido a que la saliva lubrifica los tejidos orales, limpia la boca y comienza el proceso digestivo, mientras se mastican los alimentos (2)

La saliva es un fluido primordial, porque es un mecanismo defensivo que sirve para triturar los alimentos, evitar las caries y las enfermedades periodontales. De hecho, un grupo de investigadores de la Universidad de Turku (Finlandia) ha demostrado que la saliva es capaz de mantener a raya infecciones como el herpes oral gracias a su función inmunológica (3). Además, recientemente investigaciones desarrolladas por el Instituto Pasteur (Francia) atribuyen al flujo salival propiedades calmantes y analgésicas, incluso superiores a la potente analgesia de los fármacos opiáceos como la morfina (4). Cuando el flujo salival fisiológico disminuye, también hay alteraciones gastrointestinales, y aparecen problemas para hablar, masticar, tragar y saborear los alimentos, que conducen a su vez, a trastornos de nutrición.

Las consecuencias de la Xerostomía no se limitan a estas complicaciones físicas. En algunos casos, el impacto que la enfermedad tiene sobre la vida cotidiana de los pacientes deriva en situaciones de insomnio, irritabilidad, e incluso, depresión, por eso, no es de extrañar que muchos de los enfermos vean afectada su calidad de vida desde el punto de vista de la sociabilidad, y pierdan el interés por comer en compañía, por salir o por hablar en grupo.

\section{INCIDENCIA E IMPACTO DE LA XEROSTOMÍA EN LA POBLACIÓN}

Como muestra del fuerte impacto que este trastorno supone para los pacientes y de la importancia que 
tiene el detectar, diagnosticar, tratar o prevenir la Xerostomía, Doña Ana Aliaga, Vocal Nacional de Dermofarmacia del Consejo General de Colegios Oficiales de Farmacéuticos, subrayó los resultados de un estudio realizado en forma de encuesta (extraída de artículos especializados en boca seca) en 152 Oficinas de Farmacia y un total de 563 cuestionarios recogidos, cuyo objetivo era evaluar el grado de Xerostomía o Síndrome de Boca Seca y las consecuencias de este síndrome en la calidad de vida del paciente. Los resultados demuestran que más de un $87,6 \%$ de los encuestados ante la pregunta, sobre cómo se sentirían si tuviesen que estar el resto de su vida con la sequedad de garganta que tiene ahora, se sentirían preocupados, muy descontentos u horrorizados.

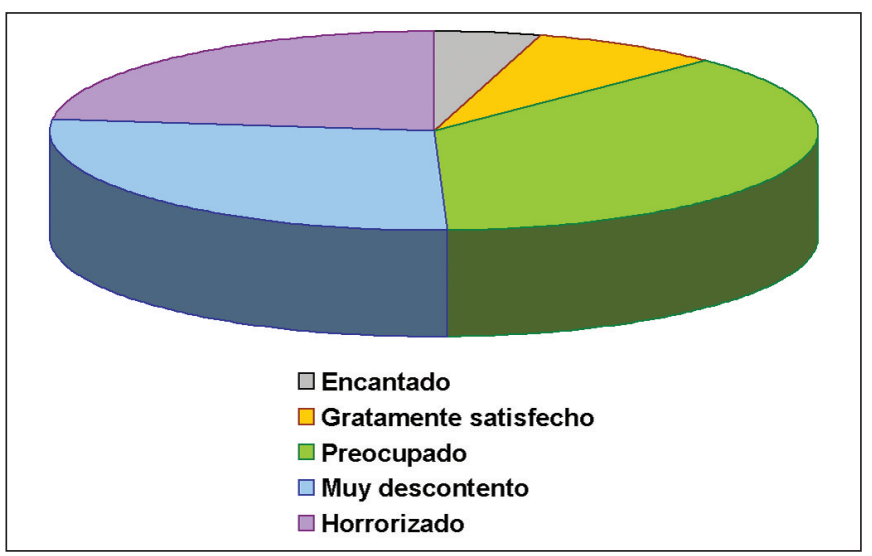

La Xerostomía o Síndrome de Boca Seca se define como la disminución del flujo salival en condiciones de reposo.

XEROSTOMIA/HIPOSIALIA
Concepto
Término clínico que traduce una hipo
o asialia.
Puede ser subjetiva u objetiva.
Se habla de hiposecreción salival
(sialopenia,hiposialia) con cifras de:
- Menos de 0,1-0,2 ml/min en reposo o menos
de 500 cc/día.
- Menos de 0,5-0,7 ml/min en estímulo.

Cortesía del Profesor Antonio Bascones, presidente de la Sociedad Española de Medicina Oral.
La Federación Dental Internacional define a la Xerostomía como "la enfermedad del hombre moderno" debido a su carácter casi epidémico.

El síndrome de la boca seca o Xerostomía afecta a una de cada cuatro personas, índice que crece a partir de los cincuenta o sesenta años. Hasta hace poco, se creía que la Xerostomía era única de las personas de la tercera edad, pero en estudios realizados en el norte de Europa, se encontró que entre el veinte y el treinta por ciento de la población, de las personas de veinte años, tienen esta patología, que puede provocar edentulismo prematuro, debido al creciente uso de antidepresivos, según el profesor Jorma Tenovuo, Catedrático de Cariología, Universidad de Turku, Finlandia. Estos datos son similares en Estados Unidos, donde hasta el cuarenta por ciento de la población puede presentar esta patología. Coincide el Profesor Antonio Bascones, presidente de la Sociedad Española de Medicina Oral, en que, el elevado consumo de antidepresivos y otros medicamentos, así como el consumo de bebidas alcohólicas y de tabaco podría explicar el aumento de personas que sufren esta alteración.

\section{LA XEROSTOMÍA Y LA GINECOLOGÍA}

La Xerostomía está presente en mayor proporción en el colectivo femenino que en el masculino, principalmente por los cambios hormonales asociados al embarazo, climaterio o a la menopausia. Hasta un ochenta por ciento de las mujeres menopáusicas padecen sequedad en las mucosas, provocada por la falta de producción de las hormonas sexuales. El Dr. Manuel Albi, Jefe de la Unidad de Ginecología y Obstetricia de la Fundación Hospital de Alcorcón, confirmó que los ginecólogos recomiendan, para paliar esta sintomatología, un tratamiento estrogénico sustitutivo, que es cuanto menos, controvertido, porque si bien puede ser efectivo para la sequedad de la mucosa vaginal, no lo es a nivel de la mucosa oral. Esto se debe principalmente, a que los receptores estrogénicos de la mucosa vaginal son de tipo alfa, en contraposición a lo receptores estrogénicos de las glándulas salivales, que son de tipo beta, y responden 
en mucho menor grado a esta estimulación estrogénica.

También se analizó la problemática del tratamiento médico para mitigar la incontinencia urinaria, por presentar como efecto secundario sequedad de boca. Si a esta circunstancia, unimos que esta patología se diagnostica principalmente en mujeres con climaterio, menopausia y postmenopausia, la sequedad bucal se agudiza de manera considerable, y está descartado por completo, el tratamiento de la misma, mediante fármacos sialogogos.

Una vez más, se comprobó que es mejor buscar alternativas en productos sustitutivos de la saliva o estimulantes de la secreción salival.

Por último, el Dr. Albi constató que existen otras patologías que producen sequedad de boca como es la diabetes de tipo gestacional, haciendo referencia al colectivo de mujeres embarazadas.

\section{LA XEROSTOMÍA Y EL ODONTÓLOGO}

En la mayoría de los casos el síndrome de boca seca cursa silente y no existe sintomatología hasta que está muy avanzado. Suelen ser pacientes que van de médico en médico y no encuentran una solución a sus problemas. Los expertos aseveran la necesidad de estar alerta, para que los facultativos puedan diagnosticarlo y prevenirlo. Por todo ello, es importante que los odontólogos presten atención a la sintomatología de los pacientes, ya que suele ser a ellos a quienes manifiesten alguna molestia, pues puede no presentarse una auténtica hiposalivación. Según algunos estudios científicos, se puede llegar a perder hasta un cincuenta por ciento del flujo salival sin todavía percibir sequedad en la boca.

La Xerostomía está asociada a problemas bucodentales graves tales como gingivitis, destrucción dental, dolor en los tejidos blandos y en la lengua, halitosis e incluso trastornos alimenticios.

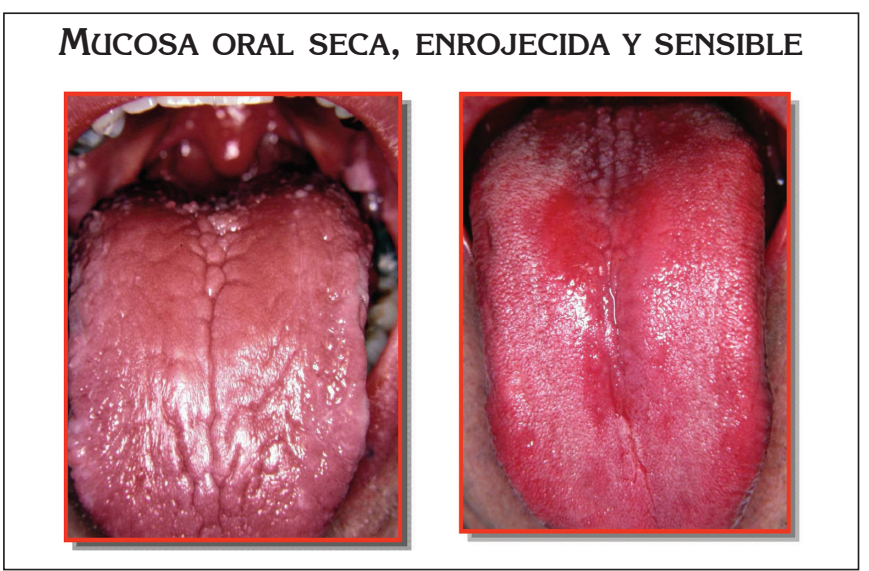

Cortesía del Profesor Antonio Bascones, presidente de la Sociedad Española de Medicina Oral.

Algunos de los síntomas más frecuentes son la sensación de ardor y dolor en la lengua, boca reseca, aparición de fisuras en las comisuras de los labios, sed constante, predominio de aftas bucales, acumulación de la placa dental bacteriana, halitosis, esmalte dental desgastado y dificultad en el uso de la prótesis dental (en el caso, de pacientes edéntulos).

Las caries constituyen uno de los principales signos de la Xerostomía, ya que son unas caries muy características, primordialmente ubicadas en las raíces de los dientes.

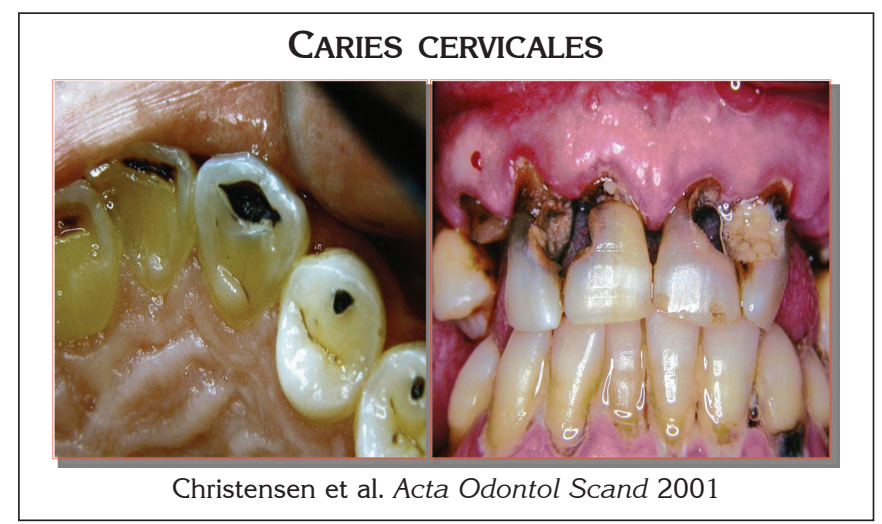

Cortesía del Profesor Antonio Bascones, presidente de la Sociedad Española de Medicina Oral.

Estas caries se producen en apenas unas semanas debido a la incapacidad por parte de la boca para mantenerse limpia, al igual que otras enfermeda- 
des periodontales, como la gingivitis. También existe un crecimiento exacerbado de la placa dental porque el flujo salival no arrastra las bacterias, y existe una menor concentración de proteínas antibacterianas.

Desde el punto de vista de los pacientes, uno de los primeros signos que denotan, es que su saliva se vuelve viscosa y espumosa.

\section{LA XEROSTOMÍA Y LA MEDICINA INTERNA}

La disminución del flujo salival se debe a una hipofunción de las glándulas salivales. Esta disminución de la cantidad de saliva puede ser reversible si la causa que la desencadena es pasajera, como un estado de ansiedad, una infección de tipo aguda en las glándulas salivales, por un estado de deshidratación o como efecto secundario de algunos medicamentos. En cambio, si la causa es de tipo crónico conduce a una sequedad de boca permanente como ocurre en algunas anomalías congénitas, en enfermedades autoinmunes, infecciosas, reumatológicas (Síndrome de Sjögren y fibromialgia), enfermedades alérgicas, menopausia, radioterapia en cáncer primordialmente de cabeza y cuello, diabetes, trastornos psiquiátricos, Alzheimer, SIDA, sustancias adictivas (alcohol, drogas y tabaco). Según recordó el Prof. Ian Mac Veigh, (Clínica Mayo Rochester. Clínica Cemtro Madrid), existen más de quinientas familias de medicamentos que inducen a una hipofunción de las glándulas salivales, como efecto adverso potencial, siendo éste uno de los principales motivos de los abandonos de la medicación por parte de los pacientes. Hay que reseñar, que estos fármacos además de presentar un elevado índice de consumo, son en su mayoría la terapia de elección en los tratamientos de enfermedades de tipo crónico o de carácter epidémico: antidepresivos tricíclicos, sedantes antihipertensivos, antihistamínicos, ansiolíticos, analgésicos, descongestionantes, diuréticos, antipsicóticos, antiinflamatorios, antiepilépticos, antiespasmódicos, antiacnéicos, antidiarreicos, relajantes musculares,...

También recordó que puede ser consecuencia del excesivo consumo de alcohol y tabaco.

\section{LA XEROSTOMÍA Y LA ONCOLOGÍA: IMPORTANCIA DE LA PREVENCIÓN}

Ciertos quimioterápicos son capaces de provocar alteraciones transitorias en el flujo salival y hasta el cuarenta por ciento de los pacientes en tratamientos del cáncer se queja de este efecto secundario. Así mismo, la radioterapia que se emplea para tratar tumores en la zona de cabeza y cuello afecta a las glándulas salivales, provocando una disminución del flujo salival. La Xerostomía está asociada a los diferentes tratamientos oncológicos, no sólo a radioterapia, a quimioterapia, sino también al trasplante de médula ósea (leucemia), los cuales cursan con severas mucositis/estomatitis, que pueden en efecto, suponer un motivo de interrupción del tratamiento oncológico.

El Prof. Eduardo Lanzós, Jefe del Servicio de Oncología del Hospital 12 de Octubre de Madrid, señaló la gran importancia de tomar todas las medidas preventivas posibles para tratar de evitar que el paciente oncológico abandone temporalmente el tratamiento, pues en ese momento, el tumor podría rebasar los límites de la curabilidad. Para conseguir este objetivo, recomendó para el tratamiento de las mucositis escoger cuidadosamente los alimentos y realizar muy bien la higiene bucal, utilizar un cepillo muy suave que evite el sangrado. Fundamental es visitar al odontólogo previamente al tratamiento oncológico, con la finalidad de eliminar cualquier viso de infecciones y caries.

Así mismo, se hizo hincapié sobre el hecho de que, en el paciente oncológico inciden además, otros factores como el nerviosismo, la irritabilidad, el insomnio o la depresión que requieren medicación con efecto xerostomizante.

\section{LA XEROSTOMÍA Y LA PSIQUIATRÍA}

La Xerostomía es un problema muy ligado a trastornos, como estrés, ansiedad y depresión, y a factores locales, como tabaquismo, alcoholismo y cafeína. En algunas enfermedades psiquiátricas, o en casos de estrés postraumático, puede tratarse de una manifestación de su enfermedad; aunque también existen personas que se quejan de síntomas orgánicos 
cuando en realidad se trata de un trastorno con un origen psiquiátrico. Estos pacientes, buscan curarse "de la boca" porque no piensan que estén enfermos de la mente.

Se hizo mención al "Tic de la deglución" dentro de los cuadros clínicos, que se caracteriza porque el paciente está continuamente tragando saliva, apareciendo tras cada trago una sensación dolorosa e intolerable. La boca se seca como consecuencia de tanto deglutir, por lo que la sensación de espasmo se intensifica. Si a esta sequedad de boca se asocia una sequedad de boca espontánea, estaríamos antes una neurosis como enfermedad del ánimo.

El Prof. López-lbor, director de la Clínica López-lbor de Madrid, señaló la alta incidencia de aparición de la Xerostomía, debido al efecto secundario de los psicofármacos utilizados en los tratamientos de las enfermedades psiquiátricas (se vio la importancia del manejo, sobre todo, de los distintos tipos de antidepresivos, con especial atención sobre los antidepresivos tricíclicos), y también, por el mecanismo intrínseco inhibitorio del propio proceso psiquiátrico. Estas circunstancias, se agravan significativamente en pacientes institucionalizados, y/o que requieran polimedicación.

Si a esto, unimos que los pacientes psiquiátricos, son remisos a cuidados y aseos personales, les convierten en los pacientes por excelencia, con la peor salud bucodental. Además, por otra parte, los pacientes con cuadros depresivos abusan de alimentos ricos en azúcares e hidratos de carbono, complicando más su sintomatología.

De nuevo, en este campo, la Xerostomía es la principal responsable del abandono de los tratamientos, por lo que es primordial utilizar un tratamiento adyuvante que minimice por completo el efecto indeseable de los psicofármacos.

\section{LA XEROSTOMÍA Y LAS PATOLOGÍAS GLANDULARES}

La Xerostomía o boca seca, también, aparece en un grupo de menor dimensión, pero no por ello, menos importante, como son los pacientes con patologías glandulares.

Es ésta, una patología con un origen multifactorial y muy complejo, que favorece la existencia de un desconocimiento, obteniéndose como resultado de manera muy frecuente el retraso en el diagnóstico y la derivación de estos pacientes de un especialista a otro, por lo que en la mayoría de los casos, no se trata de la forma más efectiva. Esta circunstancia, según coinciden los expertos, hace vital el diagnóstico precoz y un tratamiento eficaz y seguro para evitar complicaciones tales como gingivitis, la destrucción dental, el dolor en los tejidos blandos y lengua, la halitosis y, en los peores casos, los trastornos alimenticios.

\section{CÓMO DETECTAR FÁCILMENTE LA XEROSTOMÍA}

Para el diagnóstico precoz, investigadores de tres universidades americanas - Michigan, North Carolina y Nueva York, liderados por el profesor Jonathan Ship, experto internacional en Xerostomía, han diseñado una Escala Visual Analógica (6) y una Encuesta sobre la Calidad de Vida asociada al Síndrome de Boca Seca (7), cuestionario muy similar al utilizado, en la evaluación de otras condiciones subjetivas, como es el dolor. A través de este cuestionario el paciente hace una autovaloración sobre la enfermedad, en función de la cual se puede instaurar un tratamiento. En la actualidad, los tratamientos disponibles persiguen tratar los síntomas, y mejorar el flujo salival, además de prevenir las consecuencias de la sequedad de boca incluso en aquellos casos en los que no haya posibilidad de recuperar este flujo debido a una cirugía o a la administración de radioterapia para tratar tumores glandulares de cabeza y cuello.

\section{CÓMO AYUDAR A ESTOS PACIENTES}

El tratamiento de la Xerostomía incluye un seguimiento de una serie de medidas higiénico-dietéticas por parte del paciente, entre las que se incluye una mayor masticación de los alimentos para producir más saliva, subrayándose la importancia que los 
odontólogos restauren la capacidad masticatoria que han perdido estos pacientes, o la supresión de agentes que causan sequedad de boca, como el alcohol y el tabaco. Junto con estos consejos, se administran productos de higiene oral que estimulan el flujo salival. Esto, implica el uso de sustitutos salivales, saliva artificial y estimulantes salivales (sialogogos). Se señaló la capacidad estimulante de la pilocarpina, si bien se incidió en recordar aquellas patologías que contraindican su administración (pacientes asmáticos, con hipersensibilidad a la pilocarpina, en iritis aguda y en glaucoma de ángulo cerrado), así como, en sus serios efectos secundarios (cefaleas, visión borrosa, lacrimeo, hipersudoración problemas respiratorios, espasmos gastrointestinales, nauseas, vómitos, diarrea, taquicardia, bradicardia, hipotensión, hipertensión, shock, confusión mental, arritmia cardiaca y temblores).

De vital importancia resulta mantener una cuidada higiene bucal para reducir el riesgo de las infecciones y caries, tal cómo anotó el profesor Tenovuo. La saliva artificial debe poseer un $\mathrm{pH}$ neutro al igual que todos los productos utilizados en este colectivo de pacientes con grave riesgo de desmineralización. Los estimulantes salivales tales como los caramelos con o sin azúcar y ácido málico, debido a su componente acídico, pueden disolver el esmalte de los dientes. Máximo cuidado debe tenerse con las bebidas ricas en azúcares y ácidos (refrescos de naranja o limón y bebidas colas). Cuando la producción de la saliva desciende y el pH se acidifica, los iones calcio y fósforo de la saliva no pueden remineralizar el esmalte dañado y, como consecuencia de esto, aparecen caries.

Masticar chicles sin azúcar puede ser un tratamiento preferente. Así mismo, es necesario beber mucho agua, de dos a tres litros de agua diarios u otros líquidos, como son infusiones y zumos naturales.

\section{NUEVAS ALTERNATIVAS TERAPÉUTICAS}

El Prof. Michael Turner, del equipo de investigadores de la Universidad de Nueva York coordinado por el Prof. Jonathan Ship, recientemente, ha llevado a cabo un estudio a doble ciego, que demostró la potente eficacia de la combinación de aceite de oliva extra virgen, betaína y xilitol para combatir el síndrome de boca seca o Xerostomía, convirtiéndose en una alternativa más innovadora, eficaz y segura con respecto a los tratamientos actuales y tradicionales (9). Si bien, estos tres principios activos, de manera aislada, no se caracterizan por ser estimulantes salivales per sé; el uso combinado del sustituto salival, colutorio, spray y dentífrico y administrado conforme a lo establecido en el protocolo fijado por la Universidad de Nueva York, conduce a unos resultados, que despejan cualquier escepticismo, porque se aumenta hasta un doscientos por cien el flujo salival no estimulado, con tan sólo una sola semana de uso.

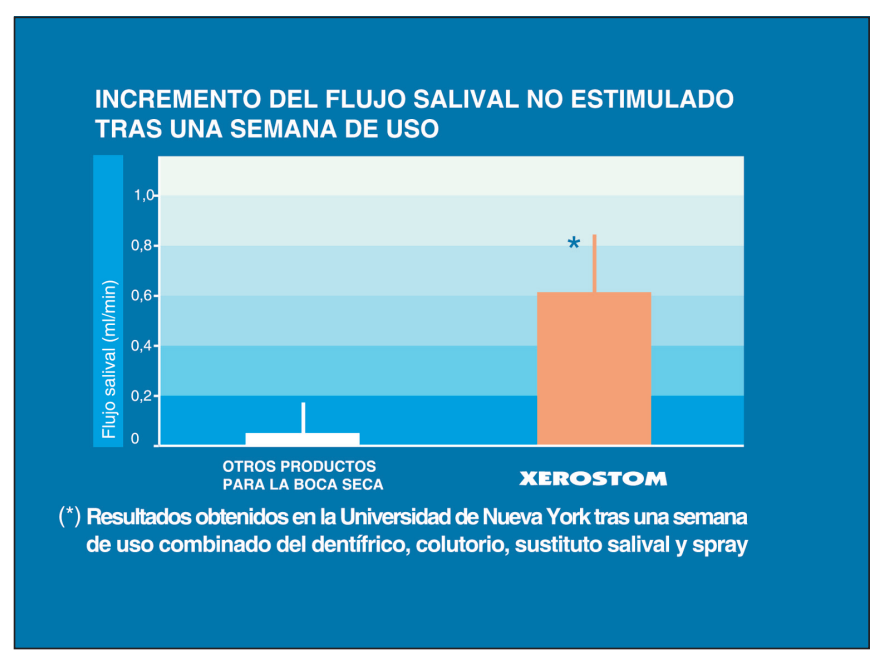

Esta combinación de principios activos, que como señaló el profesor Turner no presenta contraindicaciones, ni efectos secundarios, está incluida en la formulación de una línea comercializada bajo la marca XEROSTOM ${ }^{\circledR}$, compuesta por diferentes formas farmacéuticas de uso tópico, entre las que destacan un sustituto salival, un spray bucal, un dentífrico y un colutorio, apto en pacientes diabéticos y fenilcetonúricos. Según los expertos, esta combinación es la responsable de la reducción estadísticamente significativa de los síntomas de dolor, ardor y molestias para comer o hablar de estos pacientes. Estos buenos resultados se deben a la combinación de las propiedades antiinflamatorias, bacteriostáticas e hidratan- 
tes del aceite de oliva extra virgen, y a las propiedades antiirritantes e hidratantes de la betaína (extracto natural de la remolacha), a las que se suman las propiedades, bacteriostática y remineralizante y efecto buffer o tampón, que retorna el $\mathrm{pH}$ ácido de estos pacientes a un $\mathrm{pH}$ neutro $(\mathrm{pH}$ fisiológico).

Los resultados finales de este estudio realizado en Nueva York, presentado por el Profesor Turner, fueron:

1. Aumento hasta un $200 \%$ demostrado del flujo salival.

2. Alivio del dolor asociado a Xerostomía.

3. Mejoría importante de la sequedad de boca, lengua y garganta.

4. Mitigación de la sed por falta de saliva como consecuencia de un aumento de la saliva en reposo.

Este estudio realizado en la Universidad de Nueva York en el 2006, ha sido aprobado en el 2007 para su publicación en el "Journal of Oral Rehabilitation".

Además, la línea XEROSTOM ${ }^{\circledR}$, previene la desmineralización y destrucción dental asociada a la Xerostomía, según un estudio realizado en las Universidades de Zurich y Göttigen (10).

El principal objetivo, del Simposium 2007 multidisciplinar sobre "Xerostomía. Síndrome de Boca Seca. Boca Ardiente" celebrado en el Casino de Madrid el pasado 23 de Febrero de 2007, fue divulgar las causas, síntomas y tratamientos sobre esta patología, poco conocida entre la población, pero cada día más emergente. Con esa finalidad, se contó con la estimable colaboración de prestigiosos expertos nacionales e internacionales altamente especializados y vinculados de forma directa con la Xerostomía, que sin duda, enriquecieron el encuentro.

\section{BIBLIOGRAFÍA}

1. Ship JA, Grushka M, Lipton JA, Mott AE, Sessle BJ, Dionne RA Burning mouth syndrome: an update. J Am Dent Assoc. 1995 Jul;126(7):84253.

2. Satishchandra Pai, BDS, MDS, a Elisa M. Ghezzi, DDS, b and Jonathan A. Ship, DMD, ' Ann Arbor, Mich; Chapel Hill, NC; and New York, NY. University of Michigan, University of North Carolina, and New York University. Development of a Visual Analogue Scale questionnaire for subjective assessment of salivary dysfunction. Oral Surg Oral Med Oral Pathol Oral Radiol Endod 2001;91:311-6.

3. Tenovuo J, Antimicrobial function of human saliva-how important is it for oral health? Acta Odontol Scand. 1998 Oct;56(5):250-6.

\section{National Academy of Science 2006:10.1073.}

5. Ship JA, Pillemer SR, Baum BJ. Xerostomia and the geriatric patient.J Am Geriatr Soc 2002 Mar;50(3):535-43.

6. Cuestionario basado en la Escala Visual Analógica de Pai S, Ghezzi EM, Ship JA OOOOE 2001.

7. Encuesta sobre calidad de vida. Preserved salivary output and xerostomia-related quality of life in head and neck cancer patients receiving parotidsparing radiotherapy. Henson BS, Inglehart MR, Eisbruch A, Ship JA. Oral Oncol. 2001 Jan;37(1): 84-93.

8. Ship JA. Diagnosing, managing, and preventing salivary gland disorders. Oral Dis. 2002 Mar;8(2): 77-89.

9. Ship JA. New products for relief or dry mouth: as single blinded, open-label, cross-over study to test their efficacy. New York University. Aceptado para publicación Journal of Oral Rehabiliatation.

10. Annette Wiegand1,2, Mirja Gutsche2, Thomas Attin. Effect of olive oil and an olive oil-containing fluoridated mouthrinse onenamel and dentine erosion. Clinic for Preventive Dentistry, Periodontology and Cariology, University of Zürich. Department of Operative Dentistry, Preventive Dentistry and Periodontology Georg- 
August-University of Göttingen. Para publicar en Acta Médica 2007.

11. Narhi T. Prevalence of subjective feelings of dry mouth in the elderly. J Dent Res 1994 Jan;73(1): 20-5.

12. Sreebny LM, Schwartz SS A reference guide to drugs and dry mouth $-2{ }^{\text {nd }}$ edition. Gerodontology. 1997 Jul;14(1):33-47.
13. Protocolo de la Universidad de New York.

\section{CORRESPONDENCIA}

Biocosmetics Laboratories

Araquil, 11

28023 Madrid

Tfno.: 913571583 